\title{
Effects of Saccadic Eye Movements on Postural Control in Older Adults
}

\author{
Stefane A. Aguiar \\ Universidade Cruzeiro Sul
}

Daniela Godoi

Universidade Federal de São Carlos

José A. Barela

Universidade Cruzeiro Sul and Universidade

Estadual Paulista

\author{
Paula F. Polastri \\ Universidade Estadual Paulista \\ Renato Moraes \\ Universidade de São Paulo
}

\author{
Sérgio T. Rodrigues \\ Universidade Estadual Paulista
}

\begin{abstract}
Saccadic eye movements have been shown to affect posture by decreasing the magnitude of body sway in young adults. However, there is no evidence of how the search for visual information that occurs during eye movements affects postural control in older adults. The purpose of the present study was to determine the influence of saccadic eye movements on postural control in older adults while they stood on 2 different bases of support. Twelve older adults stood upright in 70-s trials under 2 stance conditions (wide and narrow) and 3 gaze conditions (fixation, saccadic eye movements at $0.5 \mathrm{~Hz}$, and saccadic eye movements at $1.1 \mathrm{~Hz}$ ). Head and trunk sway amplitude and mean sway frequency were measured in both the anterior/posterior (AP) and medial/lateral (ML) directions. The results showed that the amplitude of body sway was reduced during saccades compared with fixation, as previously observed in young adults. However, older adults exhibited similar sway amplitude and frequency in the AP direction under the wide and narrow stance conditions, which is different from observations in young adults, who display larger sway in a narrow stance compared with a wide stance while performing saccades. These results suggest that although older adults are affected by saccadic eye movements by a decrease in the amplitude of body sway, as observed in young adults, they present a more rigid postural control strategy that does not allow larger sway during a more challenging stance condition.
\end{abstract}

Keywords: aging, eye movements, posture, saccades, visual information

Postural control is fundamental for performing numerous daily activities. The main behavioral goals involved in the control of posture are postural equilibrium (related to the balance of forces that act on the body) and postural orientation (related to the positioning of body seg-
This article was published Online First January 26, 2015.

Stefane A. Aguiar, Institute of Physical Activity and Sport Sciences, Universidade Cruzeiro Sul; Paula F. Polastri, Faculty of Sciences, Department of Physical Education, Universidade Estadual Paulista; Daniela Godoi, Department of Physical Education and Human Movement, Universidade Federal de São Carlos; Renato Moraes, School of Physical Education and Sport of Ribeirão Preto, and Research Support Center on Chronic-Degenerative Diseases, Universidade de São Paulo; José A. Barela, Institute of Physical Activity and Sport Sciences, Universidade Cruzeiro Sul, and Institute of Biosciences, Universidade Estadual Paulista; Sérgio T. Rodrigues, Faculty of Sciences, Department of Physical Education, Universidade Estadual Paulista.

This study was supported by Fundação de Amparo à Pesquisa do Estado de São Paulo (FAPESP; process no. 2009/00899-8).

Correspondence concerning this article should be addressed to Sérgio T. Rodrigues, Laboratório de Informação, Visão e Ação (LIVIA), Departamento de Educação Física, Universidade Estadual Paulista, Av. Luis Edmundo Carrijo Coube, 14-01, Bauru, SP, CEP 17033-360, Brazil.

E-mail: srodrigu@fc.unesp.br 
ments relative to each other and the environment; Horak \& Macpherson, 1996). To achieve such purposes, the postural control system must obtain and integrate sensory cues that are provided by visual, vestibular, and somatosensory channels and use them as a basis for the production of motor activity involved in balance control (Polastri, Barela, Kiemel, \& Jeka, 2012). The fact that vision plays a fundamental role in this process is well established (e.g., Horak, 2009; Lee \& Lishman, 1975; Wade \& Jones, 1997).

Several studies have investigated the effects of eye movements on postural control in an attempt to clarify how the search for visual information that elicits such movements affects balance. In young adults, pursuit eye movements increase body oscillations associated with constantly chasing a target (Bobrova, Levik, Shlykov, \& Kazennikov, 2004; Glasauer, Schneider, Jahn, Strupp, \& Brandt, 2005). Saccadic eye movements have been shown to decrease body sway (Legrand et al., 2013; Rey, Lê, Bertin, \& Kapoula, 2008; Rodrigues et al., 2013; Stoffregen, Bardy, Bonnet, Hove, \& Oullier, 2007). Such an effect has also been observed in patients with vestibular disorders (Monzani et al., 2005) and children (Ajrezo, Wiener-Vacher, \& Bucci, 2013). Saccades are very fast eye movements that are performed to bring a new region of the visual scene to the fovea (Carpenter, 1988), which allows following objects in the environment and acquiring visual cues that might be used for the production of motor activity.

The fact that performing saccadic eye movements leads to a decrease in body sway could be attributable to an attempt to increase visual stability to perform more spatially accurate saccades that are related to the target (Stoffregen et al., 2007). Two different mechanisms have been suggested to work toward the visual stabilization of posture. The first is afferent motion perception, which uses information contained in the optic flow to minimize retinal slip in an attempt to stabilize the distance between the eye and visual scene. The second is efferent motion perception, which is based on either the copy of motor command (i.e., efference copy, meaning that the prediction of the sensory consequences of one's actions is utilized in a feedforward manner) or extraocular muscle afferents (reafferences) that are consecutive to eye movements
(Guerraz \& Bronstein, 2008). To our knowledge, no study has investigated how these processes that are involved in postural stability might be affected by the aging process.

The aging process is known to disrupt postural control, which severely increases risks of falls in older adults (Muir, Kiel, Hannan, Magaziner, \& Rubin, 2013). The mechanisms that underlie such performance decrements include deficits in sensory and motor systems (Orr, 2010; Toledo \& Barela, 2010, 2014) and limited attentional capabilities (Shumway-Cook, Woollacott, Kerns, \& Baldwin, 1997) that are acquired during the aging process. Additionally, previous studies have manipulated visual information while subjects maintain an upright stance, demonstrating that older adults have difficulties integrating visual cues into their postural control functioning. In such a case, the integration of sensory and motor systems has been shown to be affected by aging (Prioli, Cardozo, de Freitas Junior, \& Barela, 2006; Prioli, Freitas Junior, \& Barela, 2005; Wade, Lindquist, Taylor, \& Treat-Jacobson, 1995), and such differences in performance have been demonstrated under more challenging postural conditions (Prioli et al., 2006; Toledo \& Barela, 2010).

Elderly individuals also demonstrate impaired performance in eye-movement tasks compared with young adults. Generally, older adults have slower saccadic RTs (Moschner \& Baloh, 1994) and reduced accuracy of saccadic eye movements (Warren, Thurtell, Carroll, \& Wall, 2013). Paquette and Fung (2011) showed that older adults performed less accurate saccades compared with young adults under both unperturbed and perturbed standing conditions, which was attributed to subtle declines in sensorimotor integration.

Previous studies have demonstrated that manipulations of some characteristics of the optic flow can affect older adults' postural control system more intensely compared with young adults (Prioli et al., 2006; Toledo \& Barela, 2010, 2014; Wade et al., 1995), suggesting that the aging process affects how visual information is used by older adults to control balance. For this reason, investigating how the search for visual information that occurs during eye movements is affected by the aging process is fundamental. Therefore, the purpose of the present study was to inves- 
tigate the effects of saccadic eye movements on postural control performance in older adults while they stood upright in wide and narrow stances. Two bases of support were used because older adults' postural control functioning is known to be strongly affected in more challenging stances (e.g., Prioli et al., 2006). Additionally, considering older adults' impairments in the execution of saccades, two different frequencies of saccadic eye movements were analyzed to investigate whether posture is affected by the complexity of eyemovement tasks.

\section{Method}

\section{Participants}

Twelve older adults $(73.6 \pm 6.4$ years old, $156.0 \pm 5.0 \mathrm{~cm}$ tall, $64.8 \pm 10.4 \mathrm{~kg}$ body mass) participated as volunteers in the present study. The participants were recruited from a local community in the city of Bauru, SP, Brazil. Only three participants were engaged in a program of regular physical activities (i.e., hydrogymnastics at least twice per week). All of the other participants reported no regular practice of physical activity other than normal daily tasks, such as walking and housework. All of the participants had normal or corrected-to-normal vision and no knowledge of the purposes of the experiment. All of the volunteers reported no history of falls in the year before the experiment, dizziness, or postural instability, no regular incidence of back pain, no diagnosed neurological diseases, and no musculoskeletal disorder that could compromise their performance in the postural control task. The participants also did not report cognitive or memory complaints and reported no diagnosed mild or severe cognitive impairments. The participants signed a written consent, and the local University Ethics Committee approved the procedures used in this study, which were in accordance with the determinations of the Helsinki Accord.

\section{Apparatus and Procedures}

The participants were asked to stand upright, positioned $1 \mathrm{~m}$ away from a screen monitor on which the visual task was presented to them. Each trial lasted 70 s. Each participant performed two trials of the postural task in each combination of two stance conditions (wide and narrow stances) and three gaze conditions (fixation, saccades at $0.5 \mathrm{~Hz}$, and saccades at 1.1 $\mathrm{Hz}$ ), resulting in a total of 12 trials.

During the wide stance condition, the participants positioned their feet comfortably apart, at shoulder width. In the narrow stance condition, they kept their feet parallel and placed together at both the heels and toes. For the visual tasks performed in the three gaze conditions, an LCD monitor (LG, Faltron L1952H, 50/60 Hz, 0.8 A; $37.5 \times 30 \mathrm{~cm}$ ) was used, which presented a 2 $\mathrm{cm}$ diameter target represented by a circle filled in red on a white background. In the fixation condition, the target was kept in the center of the screen for the entire trial, and the participants were asked to fixate on it. The subtended visual angle of the target was approximately $1.15^{\circ}$. In the saccades at $0.5 \mathrm{~Hz}$ condition, the participants performed visually guided saccades. The target appeared first on the left side of the monitor, $9.75 \mathrm{~cm}$ away from the center, and then disappeared and reappeared immediately on the opposite side (i.e., the right side), also $9.75 \mathrm{~cm}$ away from the center. The participants were instructed to follow the target with their eyes during the entire trial. The described change in target position occurred constantly during the entire trial, with a frequency of 0.5 $\mathrm{Hz}$, resulting in a total of 35 saccadic eye movements (each target appearance lasted $2 \mathrm{~s}$ ). In the saccades at $1.1 \mathrm{~Hz}$ condition, the same visual task was used, but the frequency of horizontal saccades was $1.1 \mathrm{~Hz}$, resulting in a total of 77 saccadic eye movements (each target appearance lasted $0.9 \mathrm{~s}$ ). The total distance between the right side target and left side target for the saccades tasks was $19.5 \mathrm{~cm}$, comprising a visual angle of $11^{\circ}$ in the horizontal plane, from left to right. This angle usually requires eye movements alone (i.e., without moving the head). However, no instruction was given to the participants about head movements. No record of eye movements was used. The experimenter checked the participants' appropriate eye movements through a small camera, positioned above the monitor, to eliminate trials in which the participants did not perform every saccade required, which did not happen for any of the participants.

Before data collection, two markers were attached: one on the participants' head (posterior part, right above the occipital bone) and another 
on the participants' trunk (between the scapulae, at the eighth thoracic vertebra level). Body oscillation was measured through kinematic analysis using images recorded by two video cameras (Sony DCR DVD 205 and 405; $60 \mathrm{~Hz}$ sampling rate). APAS software (Ariel Dynamics, version 1) was used for three-dimensional reconstruction and exportation of space coordinates of the two markers in the anterior/ posterior (AP), medial/lateral (ML), and vertical directions.

\section{Data Analysis}

Although each trial lasted $70 \mathrm{~s}$, the first $10 \mathrm{~s}$ were not considered in the analyses. The data were filtered using a fourth-order Butterworth filter with a cut-off frequency of $3 \mathrm{~Hz}$. For each trial, we calculated the mean sway amplitude and mean sway frequency for the head and trunk in the AP and ML directions. The mean sway amplitude was computed as the standard deviation of positional data throughout the trial. The mean sway frequency was obtained as the sum of the product of power spectrum and frequency (Power Spectral Density, Nfft $=1024$, window $=$ Welch, noverlap $=\mathrm{Nfft} / 2$, Dflag = linear) divided by the total sum of the power spectrum, which corresponds to the center of the distribution of power across frequencies of the position time series. Variables were calculated using specific routines written in Matlab (Mathworks, version 5).

\section{Statistical Analysis}

Eight $3 \times 2$ analyses of variance (ANOVAs), with repeated measures on the two factors, were conducted to test the effects of gaze (fixation, saccades at $0.5 \mathrm{~Hz}$, and saccades at $1.1 \mathrm{~Hz}$ ) and stance (wide and narrow) on each dependent variable (sway amplitude and mean sway frequency for both head and trunk in both the AP and ML directions). Tests of contrast were conducted as post hoc tests specifically to evaluate trends in gaze effects. The significance level adopted was .05 for all of the analyses.

\section{Results}

\section{Sway Amplitude}

Anterior/posterior direction. The ANOVA revealed an effect of gaze, $F_{1.22,13.50}=6.74, p<$ $.02, \eta^{2}=.380$, on head sway amplitude. The post hoc tests of the gaze effect revealed a linear trend, showing that the head sway amplitude was higher during fixation and decreased with saccades at 0.5 $\mathrm{Hz}$ and further decreased with saccades at $1.1 \mathrm{~Hz}$. The ANOVA also revealed an effect of gaze, $F_{1.07,11.79}=7.43, p<.02, \eta^{2}=.403$, on trunk sway amplitude. The post hoc tests of the gaze effect revealed a linear trend, showing that trunk sway amplitude was higher during fixation and decreased with saccades at $0.5 \mathrm{~Hz}$ and further decreased with saccades at $1.1 \mathrm{~Hz}$.

Medial/lateral direction. The ANOVA revealed an effect of stance, $F_{1,11}=56.48, p<$ $.001, \eta^{2}=.837$, on head sway amplitude. The head sway amplitude was higher in the narrow stance condition compared with the wide stance condition. The ANOVA also revealed an effect of stance, $F_{1,11}=48.74, p<.001, \eta^{2}=.816$, on trunk sway amplitude. The trunk sway amplitude was also higher in the narrow stance condition compared with the wide stance condition (see Figure 1).

\section{Mean Sway Frequency}

Anterior/posterior direction. The ANOVA revealed an effect of gaze, $F_{2,22}=10.34, p<$ $.002, \eta^{2}=.485$, on mean head sway frequency. The post hoc tests of the gaze effect revealed a linear trend, showing that head mean sway frequency was higher during saccades at $1.1 \mathrm{~Hz}$ and decreased with saccades at $0.5 \mathrm{~Hz}$ and further decreased with fixation. The ANOVA also revealed an effect of gaze, $F_{2,22}=16.36, p<.001$, $\eta^{2}=.598$, on mean trunk sway frequency. The post hoc tests of the gaze effect indicated a linear trend, showing that the mean trunk sway frequency was also higher during saccades at $1.1 \mathrm{~Hz}$ and decreased with saccades at $.5 \mathrm{~Hz}$ and further decreased with fixation.

Medial/lateral direction. The ANOVA revealed effects of gaze, $F_{1.35,14.89}=5.08, p<.04$, $\eta^{2}=.316$, and stance, $F_{1,11}=28.20, p<.001$, $\eta^{2}=.719$, on mean head sway frequency. The post hoc tests of the gaze effect revealed a linear trend, showing that mean head sway frequency was higher during saccades at $1.1 \mathrm{~Hz}$ and decreased with saccades at $.5 \mathrm{~Hz}$ and further decreased with fixation. The mean head sway frequency was higher in the wide stance condition compared with the narrow stance condition. The ANOVA revealed effects of gaze, $F_{2,22}=4.76$, $p<.02, \eta^{2}=.302$, and stance, $F_{1,11}=23.96$, 

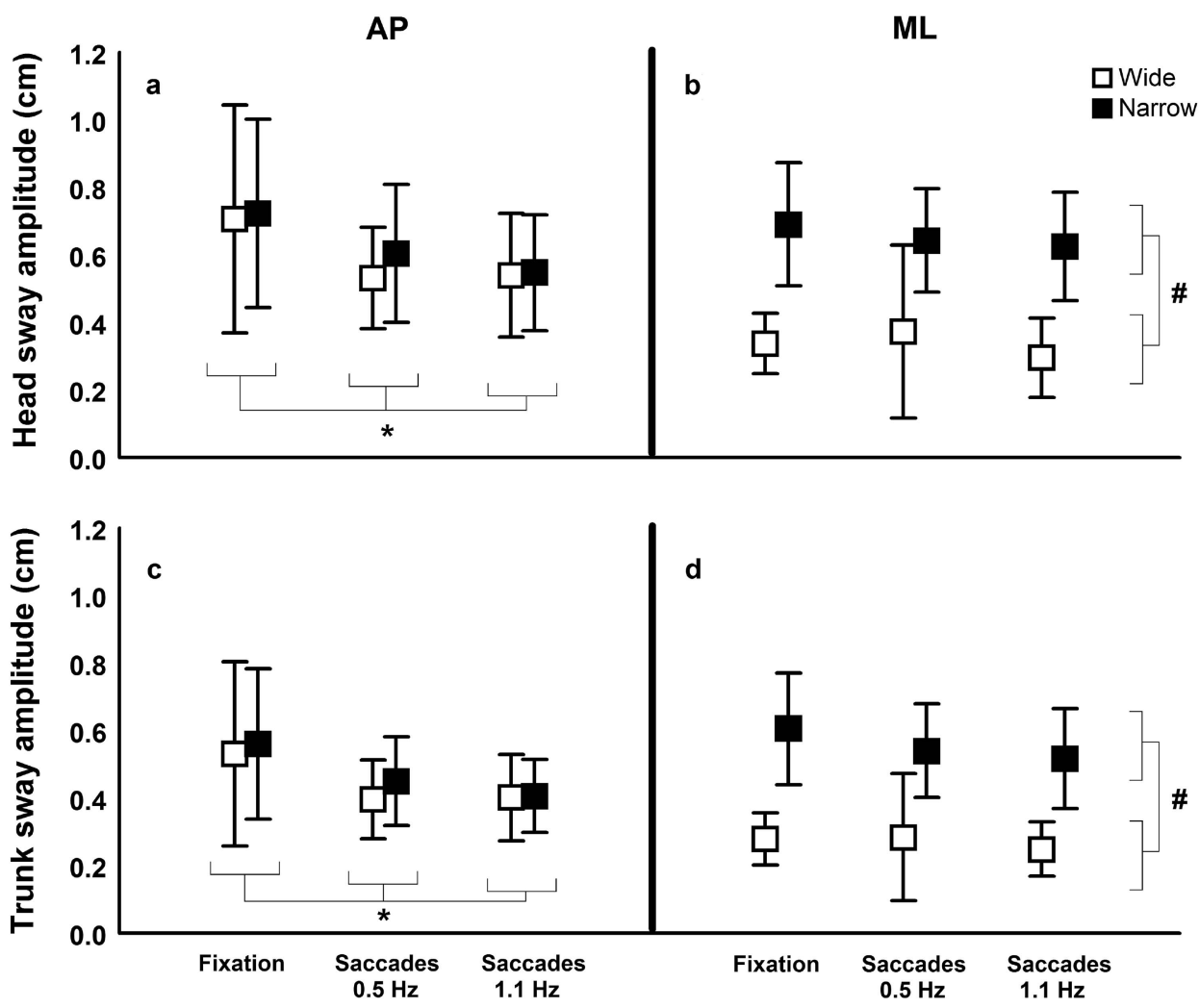

Gaze conditions

Figure 1. Head (a and b) and trunk (c and d) sway amplitude in both the anterior/posterior (AP) and medial/lateral (ML) directions during fixation, saccades at $0.5 \mathrm{~Hz}$, and saccades at $1.1 \mathrm{~Hz}$ under wide and narrow stance conditions. Error bars represent the standard deviation. \# Significant pairwise comparisons, which were used for stance effects. * Significant linear trends, which were used for gaze effects.

$p<.001, \eta^{2}=.685$, on mean trunk sway frequency. The post hoc tests of the gaze effect demonstrated a linear trend, showing that mean trunk sway frequency was higher during saccades at $1.1 \mathrm{~Hz}$ and decreased with saccades at $.5 \mathrm{~Hz}$ and further decreased with fixation. The mean trunk sway frequency was higher in the wide stance condition compared with the narrow stance condition (see Figure 2). All unreported effects did not reach statistical significance.

\section{Discussion}

The purpose of the study was to examine the effects of saccadic eye movements on postural control performance in older adults while they stood upright in wide and narrow stances. Overall, the results of the present study revealed that older adults exhibited a reduction of body sway magnitude when performing saccadic eye movements compared with fixation. However, although older adults were able to decrease body sway during saccades as previously observed for young adults, body sway was not increased in the narrow stance condition compared with the wide stance condition in older adults, contrary to young adults' behavior (Rodrigues et al., 2013). These results demonstrate a more rigid postural control strategy adopted by older adults compared with young adults, thus not allowing larger sway during a more challenging stance condition while performing saccades. 

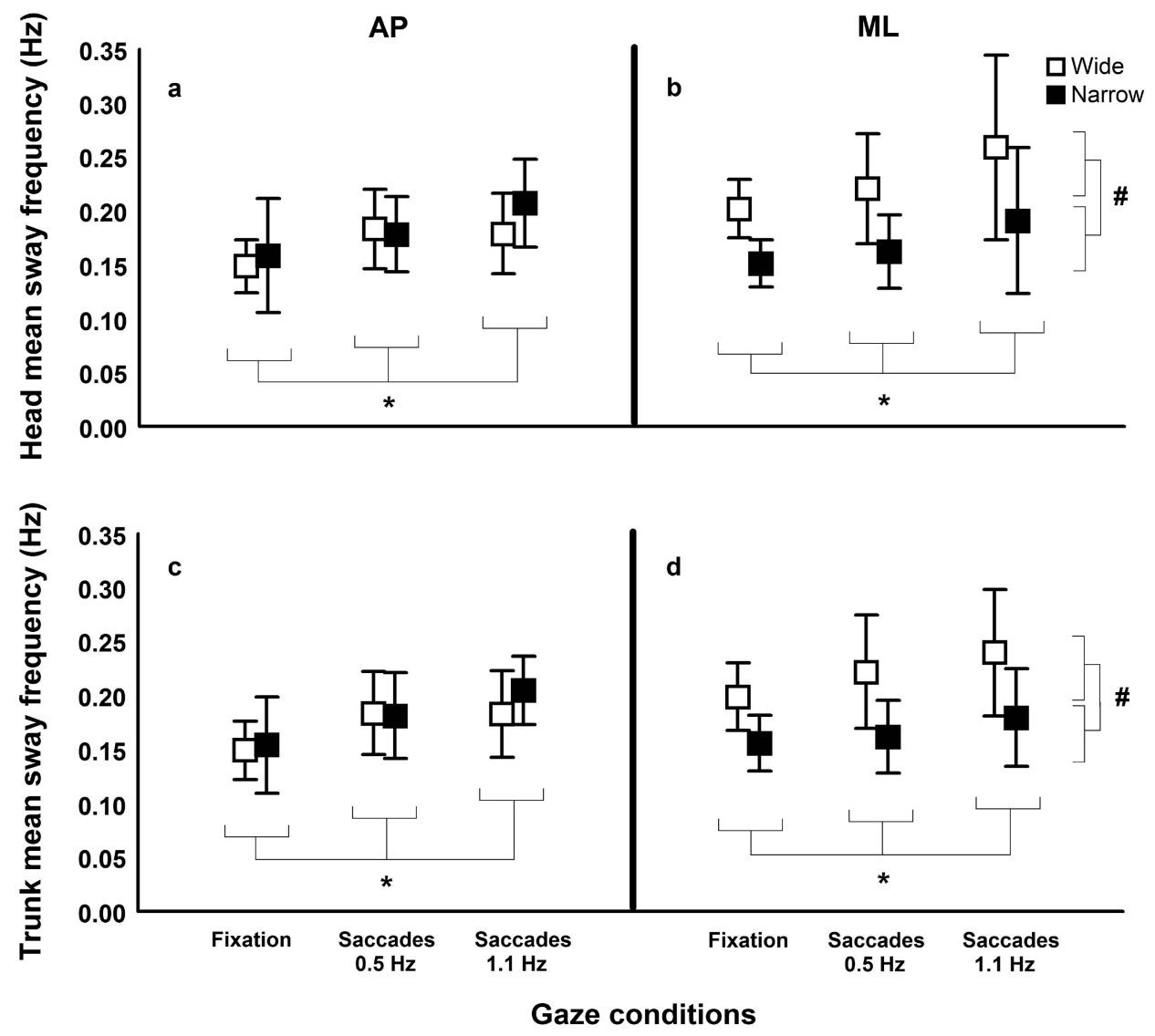

Figure 2. Head (a and b) and trunk (c and d) mean sway frequency in both the anterior/ posterior (AP) and medial/lateral (ML) directions during fixation, saccades at $0.5 \mathrm{~Hz}$, and saccades at $1.1 \mathrm{~Hz}$ under wide and narrow stance conditions. Error bars represent the standard deviation. " Significant pairwise comparisons, which were used for stance effects. * Significant linear trends, which were used for gaze effects.

Previous studies demonstrated that young adults exhibited a reduction of body sway magnitude in the AP direction and increase in mean sway frequency while performing saccadic eye movements compared with fixation. Additionally, in young adults, body sway was further attenuated in a wide stance condition compared with a narrow stance condition, especially during high-frequency saccades (Rodrigues et al., 2013). In the present study, older adults also exhibited a decrease in body sway magnitude, while increasing mean sway frequency, during saccades compared with fixation. Contrary to young adults, the present results demonstrated that the use of different bases of support in older adults did not change the body sway magnitude or frequency in the AP direction while performing saccades. In fact, the differences between the two bases of support were only observed in older adults' body sway magnitude and frequency in the ML direction, which is the direction in which body sway was expected to be affected in the narrow stance condition compared with the wide stance condition in the present study. Therefore, although young adults allow larger sway during a more challenging stance condition (i.e., narrow stance) in the AP direction, older adults did not present the same result. The demands that are involved in the wide stance condition may have already been high in older adults compared with young adults; therefore, the change to a narrow stance 
did not produce any further effects on body sway in this age group.

The fact that older adults did not allow larger sway during a more challenging stance condition while performing saccades, contrary to observations in young adults, suggests that although older adults exhibited a reduction of sway magnitude during saccades similarly to young adults, they adopted a different sway control strategy that is based on a more rigid body orientation and equilibrium to achieve such performance. Similar results were observed by Melzer, Benjuya, and Kaplanski (2001), in which older adults adopted a more rigid postural strategy through cocontraction around the ankle joint that was used in maintaining balance in a narrow stance with the addition of a cognitive task. Several other studies corroborated such findings, demonstrating an increase in muscle coactivation in the elderly compared with young adults while controlling posture (Nagai et al., 2011; Woollacott \& Shumway-Cook, 1990). The reason for the adoption of such a strategy by older adults could be attributed to a number of factors, including deficits acquired during the aging process in the sensory system, motor system, and/or sensorimotor integration processing. Importantly, a rigid postural strategy could be related to both stiffened posture caused by muscle cocontraction and a lack of flexibility to deal with such constraints as external perturbations (e.g., Mok \& Hodges, 2013; Nagai et al., 2012).

The aging process is known to compromise performance in both postural (Toledo \& Barela, 2010, 2014) and eye-movement (Warren et al., 2013) tasks when performed separately. Therefore, task demands that are involved in the visual tasks used in the present study are likely to be higher for older adults compared with young adults. For this reason, the execution of such eye-movements strongly affected older adults' performance, leading to the adoption of a rigid postural control strategy.

Such task constraints can include attentional demands. Although not examined as the focus of the present investigation, attentional aspects that are related to the execution of the eyemovement task could have influenced older adults' postural performance in the present study. Specifically, attentional demands that are involved in performing saccadic eye movements following shifts in target position are likely to be more challenging for older adults compared with young adults, which could limit attentional resources that are available for the postural control task, thus interfering with postural performance in this age group. This rationale is consistent with an adaptive resource sharing model (Mitra, 2004), which postulates that postural and suprapostural tasks compete for the same limited attentional resources. Therefore, because studies have demonstrated that older adults present limited attentional capabilities compared with young adults (Shumway-Cook et al., 1997), performing accurate saccades to reach the target could have stressed older adults' postural control system, interfered with balance, and led to a more rigid control strategy.

In the AP direction, the change to a narrow base of support compared with a wide one did not alter body sway (contrary to observations in young adults). However, in the ML direction, postural performance was changed similarly to young adults, with an increase in the magnitude and decrease in the frequency of sway with the narrow stance (Rodrigues et al., 2013). This result was expected because the narrow stance caused a reduction of the basis of support in the ML direction. With the narrow stance, the decrease in the frequency of sway that accompanies the increase in sway magnitude might be explained by the fact that reducing the basis of support led to a mechanical constraint that limited the efficiency of postural corrections in the ML direction. Such a limitation would result in a longer time that is needed for each postural correction, thus decreasing sway frequency.

One important observation in the present study was that besides the fact that saccades decreased the overall body sway magnitude compared with fixation, linear trends showed that the frequency of saccadic eye movements also played an important role in this context. Specifically, a low frequency of saccadic eye movements decreased sway compared with fixation, but saccades that were performed at a higher frequency caused a further decrease, suggesting that task demands are crucial for determining the effects of saccadic eye movements on balance control, as previously demonstrated for young adults (Rodrigues et al., 2013). This result could be attributable to the mechanisms involved in the visual stabilization of posture. Contrary to the mechanisms involved in fixa- 
tion, which are mainly based on afferent perception to try to minimize changes in the optic flow and maintain a stable relationship between the visual scene and body position, saccades are strongly based on efferent perception mechanisms. The planning of saccade execution involves a feedforward control mechanism, so the stabilization of posture could be beneficial for connecting presaccadic and postsaccadic views of the scene. During higher-frequency saccades, the time that is available to plan the execution of the next saccade movement is reduced, thus increasing task complexity, which could explain the differences in body sway between the two different frequencies of saccadic eye movements. Unfortunately, one important limitation of the present study is that no measures of eye movements were recorded, which otherwise could provide a means to discuss more specific mechanisms of the visual stabilization of posture.

In summary, the present results demonstrates that saccadic eye movements affected posture in older adults similarly to young adults by decreasing body sway magnitude compared with fixation. Nevertheless, to maintain stability similarly to young adults, the elderly needed to adopt a more rigid postural control strategy that did not allow larger sway while under a more challenging stance condition. We suggest that future studies examine the relationship between eye movements and postural control in older adults in more natural situations to asses and possibly provide a means to prevent risks of falls during daily activities that involve the search for visual information.

\section{References}

Ajrezo, L., Wiener-Vacher, S., \& Bucci, M. P. (2013). Saccades improve postural control: A developmental study in normal children. PLOS ONE, 8, e81066. http://dx.doi.org/10.1371/journal.pone .0081066

Bobrova, E. V., Levik, Y. S., Shlykov, V. Y., \& Kazennikov, O. V. (2004). Maintenance of equilibrium during tracing eye movements. Bulletin of Experimental Biology and Medicine, 138, 131-136. http://dx.doi.org/10.1023/B:BEBM $.0000048369 .53484 .4 \mathrm{~b}$

Carpenter, R. H. S. (1988). Movements of the eyes. London, UK: Pion.

Glasauer, S., Schneider, E., Jahn, K., Strupp, M., \& Brandt, T. (2005). How the eyes move the body.
Neurology, 65, 1291-1293. http://dx.doi.org/ 10.1212/01.wnl.0000175132.01370.fc

Guerraz, M., \& Bronstein, A. M. (2008). Ocular versus extraocular control of posture and equilibrium. Neurophysiologie Clinique, 38, 391-398. http://dx.doi.org/10.1016/j.neucli.2008.09.007

Horak, F. B. (2009). Postural compensation for vestibular loss. Annals of the New York Academy of Sciences, 1164, 76-81. http://dx.doi.org/10.1111/ j.1749-6632.2008.03708.x

Horak, F. B., \& Macpherson, J. M. (1996). Postural orientation and equilibrium. In L. B. Rowell \& J. T. Shepherd (Eds.), Exercise: Regulation and integration of multiple systems (series title: Handbook of Physiology, section 12, pp. 255-292). New York, NY: Oxford University Press.

Lee, D. N., \& Lishman, J. R. (1975). Visual proprioceptive control of stance. Journal of Human Movement Studies, 1, 87-95.

Legrand, A., Mazars, K. D., Lazzareschi, J., Lemoine, C., Olivier, I., Barra, J., \& Bucci, M. P. (2013). Differing effects of prosaccades and antisaccades on postural stability. Experimental Brain Research, 227, 397-405. http://dx.doi.org/ 10.1007/s00221-013-3519-z

Melzer, I., Benjuya, N., \& Kaplanski, J. (2001). Agerelated changes of postural control: Effect of cognitive tasks. Gerontology, 47, 189-194. http://dx .doi.org/10.1159/000052797

Mitra, S. (2004). Adaptive utilization of optical variables during postural and suprapostural dual-task performance: Comment on Stoffregen, Smart, Bardy, and Pagulayan (1999). Journal of Experimental Psychology: Human Perception and Performance, 30, 28-38.

Mok, N. W., \& Hodges, P. W. (2013). Movement of the lumbar spine is critical for maintenance of postural recovery following support surface perturbation. Experimental Brain Research, 231, 305313. http://dx.doi.org/10.1007/s00221-013-3692-0

Monzani, D., Setti, G., Marchioni, D., Genovese, E., Gherpelli, C., \& Presutti, L. (2005). Repeated visually-guided saccades improves postural control in patients with vestibular disorders. Acta Otorhinolaryngologica Italica, 25, 224-232.

Moschner, C., \& Baloh, R. W. (1994). Age-related changes in visual tracking. Journal of Gerontology, 49, M235-M238. http://dx.doi.org/10.1093/ geronj/49.5.M235

Muir, J. W., Kiel, D. P., Hannan, M., Magaziner, J., \& Rubin, C. T. (2013). Dynamic parameters of balance which correlate to elderly persons with a history of falls. PLOS ONE, 8, e70566. http://dx .doi.org/10.1371/journal.pone.0070566

Nagai, K., Yamada, M., Tanaka, B., Uemura, K., Mori, S., Aoyama, T., . . . Tsuboyama, T. (2012). Effects of balance training on muscle coactivation during postural control in older adults: A random- 
ized controlled trial. The Journals of Gerontology: Series A: Biological Sciences and Medical Sciences, 67, 882-889. http://dx.doi.org/10.1093/ gerona/glr252

Nagai, K., Yamada, M., Uemura, K., Yamada, Y., Ichihashi, N., \& Tsuboyama, T. (2011). Differences in muscle coactivation during postural control between healthy older and young adults. Archives of Gerontology and Geriatrics, 53, 338343. http://dx.doi.org/10.1016/j.archger.2011.01 .003

Orr, R. (2010). Contribution of muscle weakness to postural instability in the elderly. A systematic review. European Journal of Physical and Rehabilitation Medicine, 46, 183-220.

Paquette, C., \& Fung, J. (2011). Old age affects gaze and postural coordination. Gait \& Posture, 33, 227-232. http://dx.doi.org/10.1016/j.gaitpost.2010 .11 .010

Polastri, P. F., Barela, J. A., Kiemel, T., \& Jeka, J. J. (2012). Dynamics of inter-modality re-weighting during human postural control. Experimental Brain Research, 223, 99-108. http://dx.doi.org/ 10.1007/s00221-012-3244-Z

Prioli, A. C., Cardozo, A. S., de Freitas Júnior, P. B., \& Barela, J. A. (2006). Task demand effects on postural control in older adults. Human Movement Science, 25, 435-446. http://dx.doi.org/10.1016/j .humov.2006.03.003

Prioli, A. C., Freitas Júnior, P. B., \& Barela, J. A. (2005). Physical activity and postural control in the elderly: Coupling between visual information and body sway. Gerontology, 51, 145-148. http:// dx.doi.org/10.1159/000083984

Rey, F., Lê, T. T., Bertin, R., \& Kapoula, Z. (2008). Saccades horizontal or vertical at near or at far do not deteriorate postural control. Auris, Nasus, Lar$y n x, 35$, 185-191. http://dx.doi.org/10.1016/j.anl .2007 .07 .001

Rodrigues, S. T., Aguiar, S. A., Polastri, P. F., Godoi, D., Moraes, R., \& Barela, J. A. (2013). Effects of saccadic eye movements on postural control stabilization. Motriz, 19, 614-619.

Shumway-Cook, A., Woollacott, M., Kerns, K. A., \& Baldwin, M. (1997). The effects of two types of cognitive tasks on postural stability in older adults with and without a history of falls. The Journals of Gerontology: Series A: Biological Sciences and Medical Sciences, 52, M232-M240. http://dx.doi .org/10.1093/gerona/52A.4.M232

Stoffregen, T. A., Bardy, B. G., Bonnet, C. T., Hove, P., \& Oullier, O. (2007). Postural sway and the frequency of horizontal eye movements. Motor Control, 11, 86-102.

Toledo, D. R., \& Barela, J. A. (2010). Sensory and motor differences between young and older adults: Somatosensory contribution to postural control. Brazilian Journal of Physical Therapy, 14, 267-275. http://dx.doi.org/10.1590/S141335552010000300004

Toledo, D. R., \& Barela, J. A. (2014). Age-related differences in postural control: Effects of the complexity of visual manipulation and sensorimotor contribution to postural performance. Experimental Brain Research, 232, 493-502. http://dx.doi .org/10.1007/s00221-013-3756-1

Wade, M. G., \& Jones, G. (1997). The role of vision and spatial orientation in the maintenance of posture. Physical Therapy, 77, 619-628.

Wade, M. G., Lindquist, R., Taylor, J. R., \& TreatJacobson, D. (1995). Optical flow, spatial orientation, and the control of posture in the elderly. The Journals of Gerontology: Series B: Psychological Sciences and Social Sciences, 50, P51-P58. http:// dx.doi.org/10.1093/geronb/50B.1.P51

Warren, D. E., Thurtell, M. J., Carroll, J. N., \& Wall, M. (2013). Perimetric evaluation of saccadic latency, saccadic accuracy, and visual threshold for peripheral visual stimuli in young compared with older adults. Investigative Ophthalmology \& Visual Science, 54, 5778-5787. http://dx.doi.org/ 10.1167/iovs.13-12032

Woollacott, M. H., \& Shumway-Cook, A. (1990). Changes in posture control across the life span-A systems approach. Physical Therapy, 70, 799807.

Received May 13, 2014

Revision received July 30, 2014

Accepted July 30, 2014 